\title{
Index rerum ad Vol. 4
}

Abasement, 2, 4, 6, 8f. Ability (ies) -, mental, 168 -, intellectual, 112-123 Abnormal children, 57ff., 64ff., 72 f. Acceleration, 173-188 (v. menarche, menopause)

and growth, 174, 177

and illness, $178 f$.

of intelligence, 120

and lifespan, 184f. -, secular, 173f.

and socio-economic state, 175, 177 Achievement, 2, 4, 6ff.

and motor adjustment, 92 ff., 106

- $\quad$ in motor adjustment, subjective

aspects on, 90, 104ff.

- $\quad$ and repetition, 106

Adjustment

-, motor, 86ff., 96-106

- $\quad$ between sexes, 156

Adolescence, 1-13, 15-21, $30 \mathrm{ff}$.

Adulthood, 1-13, 15-21

Aggression, 2, 4, 6ff., 82

Aging, 22f., 129-133, 134f., 138, 140, 143-146 (v. brain syndrome, chronic)

and decline, 92 ff., $104 f$.

and motor adjustment, $86 \mathrm{ff}$.

and objective tests, 134-141

and sex differences, $155 f$.

in woman, 193-228 Anthropological studies, 15-21, 22-54,

77-85, 143-147, 149, 174f., 187 Attitudes, 163

towards aging, 22-54

towards danger, 220

towards children, $203 \mathrm{ff}$.

towards death, $225 \mathrm{f}$.

towards future, $196 \mathrm{ff}$.

and health, 145

towards job, 199f., 214

towards marriage, 199f., 202 ff., 206

Attitudes

-, maternal, 77-85

towards security, 222

and sex, 149, 152ff.

Behavior problems, 79 ff, 84 Birth-weight, 174

Brain syndrome, chronic, 134ff, 140f. (v. aging) 
Capacity for status, 4ff. Confession, 161 f. Conflict, 208 f., 243 Conscience, 159-165 Control, 2, 4, 6f. -, emotional, 150, 153 Culture, differences of, 17, 20, 24 ff, 77ff, 84

Decline, 92ffi, 129ff

-, psychobiologic, 134-141

Dentition, 173f.

Dependency, 240, 244

Deprivation (and adjustment to school),

229-241 Developmental 'tasks', 227 Diabetes mellitus, 138, 140f., 178 Dominance, 4ff Drive, 113 Dynamics, $168 \mathrm{f}$.

Education, 23f., 26, 28, 30ff.

-, culturally determined, 77, 83f.

Emotion (al)

problems, 80

responsiveness, 153 Escape, 2, 5ff. Expectancies, 219f.

Factor analysis, lOOff, 112f., 116ff,122

of intelligence, 116-119

of motor behavior, $102 \mathrm{ff}$.

Index rerum ad Vol. 4

251

Fears, 199ff., 215 Feelings

of autonomy, 2, 4, 6, 8f.

of failure, 130

of fulfilment, 130, 132

of resignation, $130 \mathrm{f}$.

of tolerance, 4, 8, Femininity, 4ff. Flexibility, 4, 8, 103

Geriatrics, 134ff.

Health, 143f., 146 Hospitalism, 229-241

Infant care

and behavior, $234 \mathrm{ff}$.

and mental development, $230 \mathrm{ff}$. Intelligence, 112ff. -, differenciation of, 119f. -, hypothesis of divergency in, 112-123 Interests, 143, 145f., 219

Learning, 100, 103

Leisure activities, 219f.

Life

-, fulfilment of, 129-133

-, interpretation of, 129

- $\quad$ space, $143 f$.

Maternal acceptance, 79f., 82 Maturity, 31, 33 ff., 37 Menarche, 181, 185f. Menopause, $185 f$.

Mental defective, 112f., 122 Methods:

biography, 193

clinical m., 78 f., 193

Düs fables, 242-248

interview, 193

inventories, $1 \mathrm{ff}$.

longitudinal m., 87, 194 
MLT, 136

performance test, 87-90

situational test, 79

sociogram, 145 Micro genesis, 166-171 Mongolism, 57-75

Motivation, 2, 152f., 196ff. Needs, 3, 10, 168

Old age, 46ff., 129-133, 135, 143-146

- $\quad$ and sex differences, $155 f$.

Ossification, 174, 176f.

Perception, 150, 152, 157, 166-171

Perceptual defense, 166, 170

Personality

-, consistency of, 1,11

-, development of, 15f., 20

- $\quad$ and microgenesis, 166-171

Pregnancy, 57-75

Problem children, 77-85

Recognition, 2, 5ff. Religious values, 159-165

differences, 10fE, 148-158

roles, 148f., 157 Sexuality, 154 Shock

-, emotional, 57-75

-, 'lasting', 70ff.

Sociability, 4ff., 153

Social integration, 143-147

Socialization, $5 \mathrm{ff}$.

Stress

-, emotional, $57 \mathrm{ff} .$, 67, 70, $72 \mathrm{f}$.

-, mental, 57

Succorance, 2, 5, 8f

Thematic analysis, 194ff. Time, use of, 129-133 Trait (s)

-, feminine, 148, 157 -, masculine, 148 